\title{
Calcium Channel Inhibition
}

National Cancer Institute

\section{Source}

National Cancer Institute. Calcium Channel Inhibition. NCI Thesaurus. Code C41570.

Calcium Channel Inhibition involves interference with, or restraint of, the activities of cell membrane glycoproteins selectively permeable to calcium ions. 